\title{
Towards Open-Set Text Recognition via Label-to-Prototype
} \section{Learning}

This paper was downloaded from TechRxiv (https://www.techrxiv.org).

LICENSE

CC BY-NC-SA 4.0

SUBMISSION DATE / POSTED DATE

$31-10-2021 / 02-11-2021$

\section{CITATION}

Liu, Chang; Yang, Chun; Qin, Hai-bo; Zhu, Xiaobin; Yin, Xu-Cheng (2021): Towards Open-Set Text Recognition via Label-to-Prototype Learning. TechRxiv. Preprint.

https://doi.org/10.36227/techrxiv.16910062.v1

$\mathrm{DOI}$

10.36227/techrxiv.16910062.v1 


\title{
Towards Open-Set Text Recognition via Label-to-Prototype Learning
}

\author{
Chang Liu*, Chun Yang*, Hai-Bo Qin, Xiaobin Zhu, JieBo Hou, and Xu-Cheng Yin
}

\begin{abstract}
Scene text recognition is a popular topic and can benefit various tasks. Although many methods have been proposed for the close-set text recognition challenges, they cannot be directly applied to open-set scenarios, where the evaluation set contains novel characters not appearing in the training set. Conventional methods require collecting new data and retraining the model to handle these novel characters, which is an expensive and tedious process. In this paper, we propose a label-to-prototype learning framework to handle novel characters without retraining the model. In the proposed framework, novel characters are effectively mapped to their corresponding prototypes with a label-to-prototype learning module. This module is trained on characters with seen labels and can be easily generalized to novel characters. Additionally, feature-level rectification is conducted via topology-preserving transformation, resulting in better alignments between visual features and constructed prototypes while having a reasonably small impact on model speed. A lot of experiments show that our method achieves promising performance on a variety of zero-shot, close-set, and open-set text recognition datasets.
\end{abstract}

\section{INTRODUCTION}

Text recognition is gaining popularity among both researchers and industry fellows due to its vast applications. Currently, many scene text recognition methods [2], [29], [37] have achieved promising performance on the close-set text recognition benchmarks. However, most existing methods always fail to handle novel (unseen) characters that do not appear in the training set because these methods cannot effectively map novel labels to corresponding prototypes (class centers). Consequentially, to deal with novel characters, these methods require collecting a large amount of data and retraining or fine-tuning the model. This caveat affects the applicability of these methods under the open-set recognition scenarios, in which the evaluation set contains novel characters while extra training data is inaccessible. For example, openset recognition methods can be applied to recognize minority languages, where collecting and annotating such data can be very time-consuming and expensive. Here, open-set text recognition models offer the ability to train on close but more widely used languages and then transfer to the target language. Also, these methods are feasible when input data contains constantly evolving character sets, e.g., shop names in China may contain foreign characters from Japanese.

Chang Liu, Chun Yang, Hai-Bo Qin, Xiaobin Zhu, and Xu-Cheng Yin are with the Department of Computer Science and Technology, University of Science and Technology Beijing, Beijing 100083, China (e-mail: lasercat@gmx.us; chunyang@ustb.edu.cn; 18701330507@163.com; houjiebo@gmail.com; zhuxiaobin@ustb.edu.cn; xuchengyin@ustb.edu.cn).

Corresponding author: Xu-Cheng Yin

* These authors contributed equally.
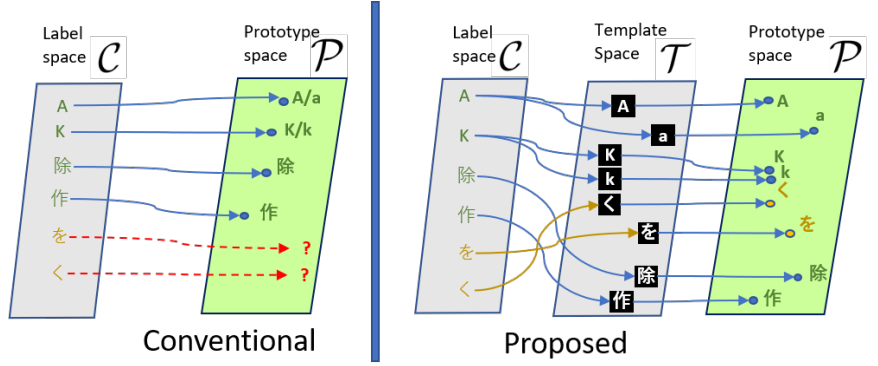

Fig. 1. The difference on prototype construction between our framework and the conventional close-set text recognition framework. Conventional methods directly construct label-to-center mapping $H$, while our proposed label-toprototype learning framework uses an intermediate temple Space $\mathcal{T}$, and decomposes $H$ into $R \circ E$ such that both $R: \mathcal{C} \rightarrow \mathcal{T}$ and $E: \mathcal{T} \rightarrow \mathcal{P}$ can be generalized on novel labels.

Conventional close-set text recognition methods fail to generalize to novel characters because they can't construct a generalizable mapping $H: \mathcal{P} \rightarrow \mathcal{C}$ that maps novel characters on the label space $\mathcal{C}$ to prototypes on the prototype space $\mathcal{P}$. More specifically, in these conventional text recognition methods, the prototypes are modeled with weights from a linear classifier, because the mapping is constructed implicitly via training, the corresponding weights for novel characters can be extremely hard to construct without retraining the model.

In the literature, a few works have been proposed to handle novel characters, which can be divided into two different schemes. One category is dedicated to handling Chinese characters. These methods map each character to a sequence of components, for example, the radical sequence of characters [3], [36]. However, mapping characters to corresponding component sequences requires strong domain knowledge. Annotating representations for each specific class is also a tedious job. Furthermore, components are mainly applicable to Chinese characters, which practically limits the feasibility in multi-language scenarios.

Another category is based on visual feature matching. Zhang et. al. [47] proposed a pipeline that first uses a siamese network to match visual features and glyphs (rendered on a single glyph-line image) into a similarity matrix, and then decodes the similarity matrix for prediction results. This pipeline does not require extensive domain knowledge against the target language and can be used for text-line recognition. However, it is less efficient against a larger charset, because it would yield a larger glyph-line image and a big similarity matrix. Additionally, the pipeline is specifically designed for text recognition 
in document images, and has limited performance on more complex text recognition tasks, e.g., scene text recognition.

To sum up, the major challenge in open-set recognition is to deal with a large number of novel classes with zero real samples. Without a generalizable label-to-prototype mapping, the conventional close-set text recognition methods always fail. Available open-set text recognition methods, however, are either language-dependent or inefficient on large character sets. Additionally, due to the unknown context information of novel characters, the context information is less applicable in openset text recognition. Thus, the robustness of visual matching becomes more important in open-set text recognition.

In this paper, to efficiently handle novel classes in a language-agnostic manner, we propose a label-to-prototype learning framework, where a label-to-prototype mapping module is learned (see Fig. 1) to generate character prototypes for the classifier. This module first maps labels (characters) to corresponding templates in the template space $\mathcal{T}$ via a font render $R$, and learns a generalizable function $E$ for mapping templates to the prototype space. The generated prototypes are then utilized by the open-set predictor as classifier weights.

Moreover, we propose a feature-level rectification technique in the feature extractor module of the recognition backbone with topology-preserving transformation, where most characters would be valid after rectification as long as the topology relationships among all its strokes are preserved. Our approach keeps the recognition engine small and fast by avoiding dedicated rectification networks [1], [21], [41].

Finally, a variety of experiments are conducted and analyzed. We first compare our method with state-of-the-art methods on two sets of well-recognized benchmarks. The first is zero-shot Chinese character recognition. Our proposed framework shows good generalization capability against novel characters. On the CTW dataset [44], the character recognition accuracy (with 2000 characters in training) is improved by $13.98 \%$ compared with a recent method [3]. The second is close-set scene text recognition. Experiments show that our method suffices as an applicable and general light-weight text recognition method. These two challenges can be regarded as special cases of the proposed open-set text recognition task. Finally, we construct a new open-set text recognition dataset and perform various comparative experiments. Our proposed method shows promising performance in open-set scenarios.

In summary, our main contributions are:

- A novel label-to-prototype learning framework is introduced for open-set text recognition. The proposed framework can handle open-set text recognition with a large charset, while keeping reasonable speed and competitive performance on conventional benchmarks.

- A topology-preserving transformation technique is proposed for text recognition which performs fast finegrained rectification alongside the feature extraction process.

- A new open-set text recognition dataset is constructed which includes a large character set and complex deterioration images from the real world. Moreover, the codes of our method and the dataset are made publicly available ${ }^{1}$

\section{RELATED WORK}

\section{A. Text Recognition}

Most conventional text recognition methods can fit into the four-stages flow [1], i.e., transformation (Trans.), feature extraction (Feat.), sequence modeling (Seq.), and prediction (Pred.). The Trans. stage is optional. In [1] and [28], a spatial transformer network is used for automatic rectification. And other methods [41] construct heavy neural networks to generate the control points and conduct image transformation. The Feat. stage generally uses a convolutional network, e.g., ResNet [12], RCNN [14], and VGG [22], to extract visual features from the rectified images. The Seq. stage captures the contextual information of the whole sequence, by using LSTM layers [4], [27], transformers [15], or simple rules [2], [27], [37]. For the Pred. Stage, several typical techniques can be used, e.g., RNN-Attention decoder, LSTM-CTC predictor [27], and decoupled text decoder [37]. Towards open-set text recognition, our proposed method extends the four-stage flow with label-to-prototype mapping and open-set prediction.

\section{B. Generalized Few Sample Learning}

Generalized Few Sample Learning [20] is an extension to existing few-shot learning tasks for addressing the forgetting issue. This task is mostly addressed with data augmentation [11], [39], and weight imprinting based methods [7], [8], [9], [24], [25], [42]. Different from conventional fewshot learning problems, the open-set text recognition task requires handling both samples from the base (seen) categories and novel (unseen) categories. Among current methods, the imprinting-based methods fit well for the openset text recognition tasks. Because imprinting-based methods usually don't require exploiting relationships among classes, the computation cost will not increase quadratically as the label set grows. These methods first generate the prototypes of unseen classes from samples or attributions. Then they concatenate generated prototypes with the prototypes from seen classes and use them as weights of the linear classifier. The imprinting-based methods also demonstrate the capability to align different domains, and can be applicable to zeroshot learning tasks [7]. However, these methods focus on the recognition of the individual objects and are not designed to handle sequences like text lines. Our label-to-prototype learning framework is closely related to imprinting-based zeroshot learning methods. Instead of generating prototypes from real samples, our method utilizes glyphs from the Noto fonts by mainly focusing on intra-class variance caused by different "cases" of characters, and largely reduces training complexity.

\section{Few/Zero-Shot Character Recognition}

Most existing few/zero-shot character recognition methods focus on English character recognition. More complicated methods are necessary for the zero-shot Chinese character

${ }^{1}$ https://github.com/lancercat/OSOCR 


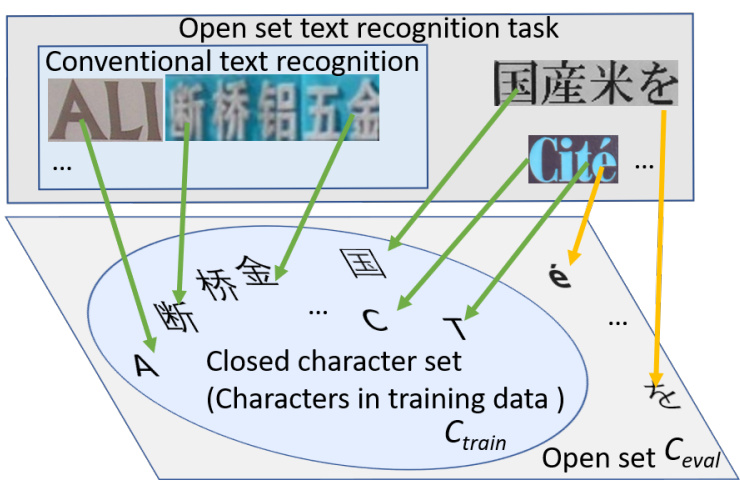

Fig. 2. Open-set text recognition task: During the evaluation process, some samples may include characters not appearing in the training set. The task requires the model to recognize both seen characters (green arrows) and novel characters unseen in the training set (yellow arrows).

recognition tasks [3] because of the large charset and high inter-class similarity. However, some available methods [36], [3] require prior knowledge of the language, where detailed annotations on the composition of radicals of each character should be provided. These methods are challenging for Chinese character recognition and text (character sequence) recognition. Recently, Zhang et al. [47] proposed a visualmatching-based method which can perform recognition on novel characters. However, this method would yield much computation burden during training for encoding "glyph-line image" with a large charset. Our proposed method only takes templates from the Noto fonts and does not need any domain knowledge of the specific language.

\section{PRoposed Method}

\section{A. Problem Formulation}

As few works formally formulate the open-set text recognition (OSTR) task, we define the open-set text recognition task as follows (Fig. 2). Given the charset of the training set $C_{\text {train }}$ and the charset of the testing set $C_{\text {eval }}$, the OSTR task assumes that $\left|C_{\text {eval }}-C_{\text {train }}\right| \geq 0$, which means novel characters (unseen characters) may appear in evaluation. Conventional close-set text recognition, in contrast, requires no novel characters to appear. Hence, it can be regarded as a special case when $\left|C_{\text {eval }}-C_{\text {train }}\right|=0$. Zero-shot learning character recognition [3], [36] is also a special case of the OSTR task when $C_{\text {eval }} \cap C_{\text {train }}=\varnothing$, and all samples have constant length 1 , as each individual character can be considered as a string of length 1 . Unlike zero-shot single character recognition and conventional close-set text recognition with many standard benchmark datasets, the general OSTR task is still rarely studied.

Generally speaking, the open-set text recognition task is to perform text recognition with potentially novel characters that do not appear in the training set (Fig. 2). Different from the generalized zero-shot learning from image classification [23], our task does not require $C_{\text {train }} \subset C_{\text {eval }}$, as character sets of different languages may not always show inclusive relations. For example, Japanese and Chinese share some characters in common, but each language has its unique characters. Here, we propose a novel open-set text recognition challenge (dataset) to validate our method. The proposed dataset uses Chinese scene text images for training while Japanese text images for evaluation. The dataset is prepared based on existing openaccess datasets. The scripts used to prepare the dataset are also released alongside our codes. For performance metrics, we adopt the popular line accuracy (LA) 2 [1] and character accuracy (CA) 3 [5] criterion commonly used in the OCR community. More details can be referred to Section IV-D1.

\section{B. Framework Overview}

In this work, we propose a label-to-prototype learning framework (Fig. 3) to address two major challenges in open-set text recognition, i.e., large charsets with novel characters and robust visual feature matching. To keep evaluation (inference) fast, we choose an RNN-free text recognition backbone in our framework for less computation cost. We adopt a modified decoupled attention network (DAN) [37] as the backbone of our framework. More specifically, we remove the RNNs from DAN for speed-accuracy trade-off reasons. Also, other available text recognition methods are also easily used as the backbone. The procedure of our framework is as follows: We first extract the rectified feature map for the images in the batch, where a topology-preserving transformation network (TPTNet) is proposed to rectify shallow visual features and to improves the robustness of visual similarity in open-set text recognition. We then serialize the extracted feature map with a convolution attention module [37] into a feature sequence $F$, where each location (timestamp) corresponds to a character in the corresponding image clip.

Then, the label-to-prototype learning module maps each character in the sampled charset $\mathcal{C}$ to corresponding (multicenter) prototypes $P$. The module is introduced to provide a generalizable label-to-prototype mapping that applies to both seen and novel characters. During the evaluation phase, $P$ can be cached for the dataset as the mapping $H$ does not change. Thus, our pipeline does not yield significant overhead during evaluation. Next, an open-set predictor is constructed to generate similarity scores $A_{t} \in R^{|\mathcal{C}|+2}$ at timestamp $t$ according to feature $F_{t}$.

During training, a label sampler module is used to sample a subset $\mathcal{C}_{\text {batch }}$ from the full charset $\mathcal{C}_{\text {train }}$ according to the labels of images in the current batch. The sampler is proposed to keep the computation cost reasonable while training with large charsets. The evaluation process is slightly different from the training process with two aspects.

\section{Topology-Preserving Transformation Network}

Scene text images are prone to different shape distortions, e.g., rotations, perspective transformations, and complex irregular distortions. These shape distortions make aligning the

\footnotetext{
${ }^{2}$ The line accuracy is also known as word accuracy (WA), same to 1-WER (Word Error Rate).

${ }^{3}$ The character accuracy is also known as accuracy rate (AR), same to 1-NED (normalized edit distance).
} 


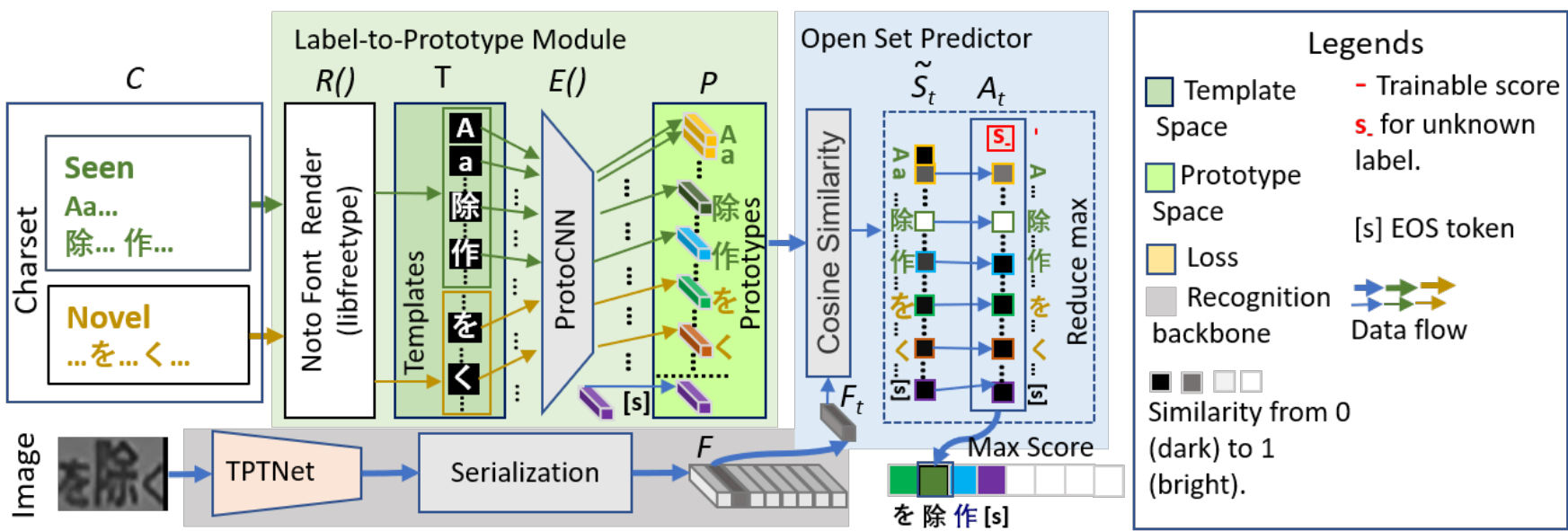

Fig. 3. Evaluation phase of our proposed framework. Image feature is extracted and serialized with the text recognition backbone, then novel and seen characters are mapped to corresponding prototypes via the label to prototype module. Finally, the open-set predictor computes the similarity $\tilde{S}_{t}$ between the extracted feature $F_{t}$ and prototypes $P$ and then reduces to character score vector $A_{t}$. Note $P$ can be cached for the dataset so the label-to-prototype module does not cause significant overhead.

prototypes and visual features difficult. To improve the robustness for visual similarity matching, we propose a topologypreserving transformation network (TPTNet) which adaptively performs a dense mesh grid transformation for rectifying feature maps. Unlike other rectification methods [21], [41], our rectification module is embedded into the feature extractor for faster speed. The process of transformation parameters estimation shares most computation with the feature extractor, thus our method can avoid the cost of a dedicated rectification network. We fuse the topology-preserving transformation (Figure 44) into the first block of ResNet, yielding the TPTNet.

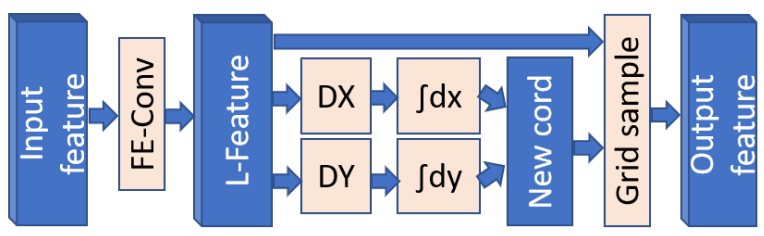

Topology-preserving transformation block

Fig. 4. Topology-preserving transformation: Each input feature map is first processed into the local feature map $M_{l}$ with a convolutional block (FEConv). Next, we estimate the effective feature density with $D X$ and $D Y$ modules. Then, we integrate the density into destination coordinates. Finally, we rectify $M_{l}$ with a grid sampler according to the destination coordinates.

The topology-preserving transformation takes a feature map $M_{i}$ as input, and produces a processed and rectified feature map $M_{o}$ as output. More specifically, we first process the feature map into a local feature map $M_{l}$ with a convolutional block,

$$
M_{l}=\operatorname{Conv}\left(M_{i}\right),
$$

Next, we estimate the feature density $D x$ on the $X$ direction and $D y$ on the $Y$ direction with corresponding $1 \times 1$ convolutional operations (see $D X$ and $D Y$ in Fig. 4) with the following activation function, i.e.,

$$
A(x)=\frac{e^{x}}{e^{x}+1}+b,
$$

where $b$ interprets as "hardness" and controls the extent of distortion what the module can perform. Formally, the feature density is estimated via

$$
\begin{aligned}
D x & =D X\left(M_{l}\right) \\
& =A\left(\operatorname{Conv}_{x}\left(M_{l}\right)\right), \\
D y & =D Y\left(M_{l}\right) \\
& =A\left(\text { Conv }_{y}\left(M_{l}\right)\right) .
\end{aligned}
$$

We then perform integration on the feature density and normalize the resulting coordinates by

$$
\begin{gathered}
w_{h, w}^{\prime}=w \frac{\sum_{i=0}^{w} D x_{h, i}}{\sum_{i=0}^{W} D x_{h, i}}, \\
h_{h, w}^{\prime}=h \frac{\sum_{j=0}^{h} D y_{j, w}}{\sum_{j=0}^{H} D y_{j, w}},
\end{gathered}
$$

where $D x_{h, i}$ indicates the element at the $h$-th row and $i$ th column of matrix $D x$, and the same goes with $D y_{j, w}$. Finally, we rectify the local features $M_{l}$ according to the coordinates $\left[w^{\prime}, h^{\prime}\right]$ into the output features $M_{o}$ using a linear sampler. As the density is always greater than zero, the integration operation is able to preserve the input topology. The gradients of coordinates are computed similar to the deformable convolution network in [6].

\section{Label-to-Prototype Learning}

1) Label-to-Prototype Learning Module: The key point of open-set text recognition is to construct the mapping $H$ from the label space $\mathcal{C}$ to the prototype space $\mathcal{P}$ that can be applied to both seen characters and novel characters. For conventional models, the mapping is implicitly applied during the training process by hashing each character to a row of the classifier weights. However, the hashing function is left 
unknown. Hence, for novel characters, such methods have to collect data and retrain the model. To construct a mapping function for both seen and novel characters, we introduce an intermediate template space $\mathcal{T}$, where its elements are $32 \times 32$ binary images. Formally, for character $C_{i} \in \mathcal{C}$, there are

$$
\begin{aligned}
\mathcal{T}_{i} & =R\left(C_{i}\right), \\
\mathcal{T} & =\cup_{i} \mathcal{T}_{i}, \\
P_{j} & =E\left(T_{j}\right), T_{j} \in \mathcal{T} .
\end{aligned}
$$

Here, $R: \mathcal{C} \rightarrow \mathcal{T}$ maps each character to all its cases. Then each case is represented by the corresponding template generated with the Noto-font ${ }^{4}$. "Case" here refers to different forms of a character, e.g., upper-case and lower-case, simplified Chinese and traditional Chinese, Hiragana and Katakana, and different contextual shapes. As Noto-font covers most characters in many languages, $R$ is highly likely to generalize to novel characters not appearing in the training set, and $R$ requires little prior knowledge of the specific languages.

$E: \mathcal{T} \rightarrow \mathcal{P}$ learns the mapping from the template space to the prototype space with the prototypical convolutional neural network (ProtoCNN). The ProtoCNN is composed of a ResNet18 network and a normalization layer. Since character frequency on the training set is potentially biased, we apply normalization on the generated prototypes to alleviate this effect:

$$
\begin{aligned}
P_{j}^{\text {raw }} & =\operatorname{ResNet}\left(T_{j}\right), \\
P_{j} & =\frac{P_{j}^{\text {raw }}}{\left|P_{j}^{\text {raw }}\right|} .
\end{aligned}
$$

We assume that the templates of seen characters fill the template space $\mathcal{T}$ densely enough, so $E$ can be generalized to novel classes. In addition, we use trainable parameters with random initialization in the prototypes for special tokens that do not have a corresponding shape, e.g., the EOS token. We also propose a regularization term $L_{e m b}$ to improve the margin between prototypes, which is described in more detail in section II-E2

During the evaluation, we can cache all prototypes because the parameters are no more updated. Thus, the label-toprototype learning module only causes little increased computation during evaluation.

2) Open-Set Predictor: An open-set predictor module is constructed to compute the similarity between serialized visual features and each character in the charset according to the corresponding prototypes. More specifically, We first compute similarity scores $\tilde{S} \in R^{T \times N}$ by multiplying the trainable margin $\alpha$, the visual feature $F \in R^{T \times D}$, and the normalized prototype matrix $P \in R^{D \times N}$ as

$$
\tilde{S}=\alpha F P .
$$

Here, the similarity score of the feature at time $t$ and the $n$-th prototype can be written as:

$$
\begin{aligned}
\tilde{S}_{t, j} & =\alpha F_{t} P_{j} \\
& =\alpha\left|P_{j}\right|\left|F_{t}\right| \cos \left(P_{j}, F_{t}\right) \\
& =\alpha\left|F_{t}\right| \cos \left(P_{j}, F_{t}\right), \quad \forall j\left|P_{j}\right|=1 .
\end{aligned}
$$

${ }^{4}$ The Noto fonts for most languages are available at https://noto-website-2. storage.googleapis.com/pkgs/Noto-unhinted.zip
Since $\alpha\left|F_{t}\right|$ is a constant number given a certain timestamp $t$, $\tilde{S}_{t}$ can be interpreted as scaled cosine similarities between all prototypes in $P$ and visual feature $F_{t}$. Hence, $\tilde{S}$ is detonated as the similarity score. Also, $\left|P_{j}\right|$ can be interpreted as the overall "preference" of prototype $j$ as it is irrelevant to the visual feature $F_{t}$. We argue the biased character frequency on the training set may affect $\left|P_{j}\right|$ and cause overfitting, so we conduct normalization on prototypes via Eq. 6 .

We then apply max reduction on $\tilde{S}$ according to labels into the classification scores $\tilde{A} \in R^{T \times(|\mathcal{C}|+1)}$, i.e.,

$$
\tilde{A}_{t, i}=\max _{\{j \mid \phi(j)=i\}}\left(\tilde{S}_{t, j}\right)
$$

where $\phi(j)$ is the label function mapping all $N$ prototypes to $|\mathcal{C}|+1$ labels, and the extra label comes from the EOS token. This strategy allows us to assign corresponding centers to different cases for characters, e.g., 'A' and 'a' with case agnostic annotations.

Given the ability to reject other "unknown" characters not covered in $\mathcal{C}_{\text {eval }}$, we adopt a trainable similarity threshold $s_{-}$ against all known labels. Because "unknown" is a result of not similar to any characters in the charset rather than sharing a common center, we model it as a trainable score instead of a prototype like the EOS token' $[s]$ '. In fact, "unknown" characters are not similar to any characters or tokens in $\mathcal{C}$, while potentially come from different centers. The score is added to $\tilde{A}_{t}$ at each timestamp $t$, yielding the final score vector $A_{t} \in R^{(|\mathcal{C}|+2)}$ :

$$
A_{t}=\left[\tilde{A}_{t, 1}, \tilde{A}_{t, 2}, \ldots, \tilde{A}_{t,|\mathcal{C}|+1}, s_{-}\right]
$$

\section{E. Optimization}

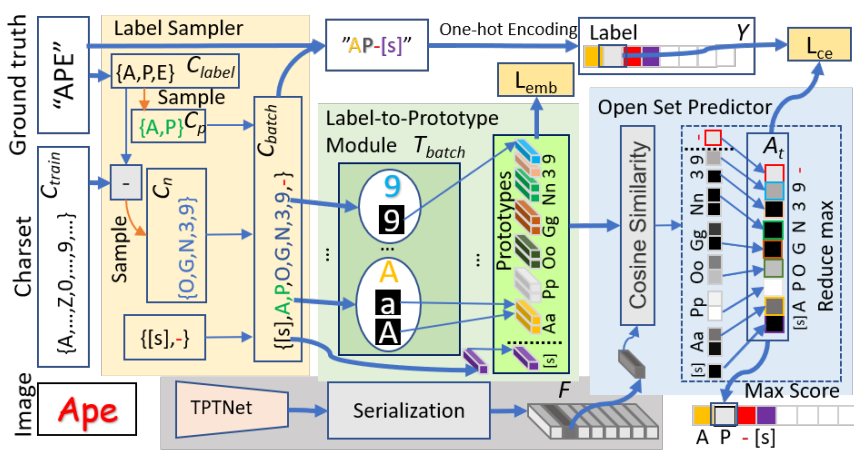

Fig. 5. The training phase of our proposed framework. We use a label sampler to keep the training complexity under control as prototypes cannot be cached due to the constantly changing label-to-prototype module. The regular term $L_{e m b}$ is also proposed to improve prototype distribution on the prototype space $\mathcal{P}$.

To control training complexity, we adopt a label sampler during the training process to reduce the training cost of the label-to-prototype module. We also propose a regularization term $L_{e m b}$ to improve the prototype distribution. The full training pipeline is illustrated in Fig 5 
1) Label Sampler: Due to the potentially large character set in Asian languages, it is computationally expensive to generate prototypes for all characters in each training iteration. In our method, we sample a subset $\mathcal{C}_{\text {batch }}$ from the full charset $\mathcal{C}_{\text {train }}$ in each iteration with the label sampler. The sampler is proposed to ensure coverage of both positive (characters that appear in the images), negative samples (characters that do not appear), and unknown samples (characters that appear but not sampled). In practice, instead of sampling from the label space, we sample from the template space to achieve more flexibility.

For a specific iteration, the character $\mathcal{C}_{\text {batch }}$ is composed of three subsets: the "positive" characters $\mathcal{C}_{\text {pos }}$, the "negative" characters $\mathcal{C}_{n e g}$, and the special characters $\{[s],-\}$. We first collect all characters that appear in labels, denoted as $\mathcal{C}_{\text {label }}$. Then, we sample a fraction of the $\mathcal{C}_{\text {label }}$ as the "positive" subset $\mathcal{C}_{\text {pos }}$. We randomly sample $\mathcal{C}_{\text {pos }}$ following two conditions: first, $\left|\mathcal{C}_{\text {pos }}\right| \leq\left|\mathcal{C}_{\text {label }}\right| * f_{s}$, and all prototypes corresponding to $C_{\text {pos }}$ should be smaller than the batch size limitation $n_{\max }$. Here the remaining characters in $\mathcal{C}_{\text {label }}-$ Cpos are considered as the "unknown" label '-' for the iteration. This approach helps training of the unknown score $s_{-}$. To cover more characters on the label space $\mathcal{C}$, we randomly sample "negative" characters $\mathcal{C}_{n e g}$ that don't correspond to characters that appear in the labels $\left(\mathcal{C}_{\text {train }}-\mathcal{C}_{\text {label }}\right)$. We also limit it's size to $\left|\mathcal{C}_{\text {neg }}\right|=\min \left(n_{\text {max }}-\left|\mathcal{C}_{\text {pos }}\right|,\left|\mathcal{C}_{\text {train }}-\mathcal{C}_{\text {pos }}\right|\right)$ to keep the computation cost under control. We then merge $\mathcal{C}_{\text {pos }}, \mathcal{C}_{\text {neg }}$ and special labels into the final charset $\mathcal{C}_{\text {batch }}$. The process is illustrated in Fig. 5 in details.

2) Regularization on Prototypes: A regularization term $L_{e m b}$ is adopted to improve the margins between classes. More specifically,

$$
\begin{aligned}
O & =P^{T} P, \\
L_{e m b} & =\sum_{i, j}^{N, N} \operatorname{Relu}\left(O_{i, j}-m_{p}\right),
\end{aligned}
$$

where $m_{p}$ is the maximum cosine similarity among two distinct prototypes for $N$ evenly distributed prototypes on a $D$ dimension space. "Evenly distributed" means the distance between each prototype and its nearest neighbor equals to the same number. Here, the margin, $m_{p}$, is used to preserve space for novel classes, and can be approximately solved via gradient descent. We estimate $m_{p}$ by optimizing the following formula:

$$
\begin{aligned}
& Q^{*}=\arg \min _{Q} \max _{(i, j)}\left(Q^{T} Q-2 J\right)_{i, j} \\
& m_{p}=\max _{(i, j)}\left(Q^{* T} Q^{*}-2 J\right)_{i, j}
\end{aligned}
$$

where $Q$ is a $D \times N$ matrix with the random initialization, each column in $Q$ is a unit vector, $N$ is the estimated number of prototypes of the open set, $D$ is the dimension of the prototype, and $J$ is an identity matrix. In this work, $D$ is set to 512 and $\mathrm{N}$ to 50,000 . That is to say, we estimate character space $\mathcal{C}$ contains 50, 000 characters. And optimization result of Eq. 12 suggests $m_{p}$ is around 0.14 .
3) Objective Function: In our system, we employ the crossentropy loss $L_{c e}$ for the classification task,

$$
L_{c e}=-\frac{1}{T} \sum_{t}^{T} \log \left(\sum_{i}^{|\mathcal{C}|+2} \frac{Y_{t, i} e^{A_{t, i}}}{\sum_{j}^{|\mathcal{C}|+2} e^{A_{t, j}}}\right),
$$

where $Y_{t} \in\{0,1\}^{1 \times(|\mathcal{C}|+2)}$ is the one-hot encoded label at timestamp $t$, and operator $*$ indicates scalar multiplication.

The object function $L_{\text {model }}$ is the combination of the classification loss $L_{c e}$ and the regularization term $L_{e m b}$, and there is

$$
L_{\text {model }}=L_{c e}+\lambda_{e m b} L_{e m b} .
$$

\section{EXPERIMENTS}

We conduct a variety of experiments to validate the proposed framework on three tasks. First, experiments on zeroshot Chinese character recognition provide a referenced comparison on the capability of our method to handle novel characters. Then, our model is tested on standard closeset text recognition benchmarks to validate its applicability in conventional applications, where conventional state-of-theart close-set text recognition methods are compared. Finally, specific experiments of our proposed framework on openset text recognition are conducted and analyzed. Experiments show that our label-to-prototype learning framework can adapt to all three tasks without modification. Also, ablative studies are conducted to validate the effectiveness of the proposed modules in different tasks.

\section{A. Implementation Details}

Our system is implemented on Pytorch, and all experiments can be trained on Nvidia GPUs with 8GB memory or larger. Codes for our method and dataset are publicly available 5

The structure of TPTNet is similar to the ResNet45 network [37], except that we replace the first layer with our TPT block. The hardness $b$ of TPTNet is set to 0.5 to limit the extent of spatial transformation for reducing training variance. The backbone for prototype generation is a standard 18-Layer ResNet, while the open-set predictor does not introduce extra hyper-parameters. In the label sampler, $f_{s}$ is set to 0.8 , and $n_{\max }$ is set to 512 . For the loss, $\lambda_{e m b}$ is set to 0.3. $\max T$ is set 2 for zero-shot Chinese character recognition ( IV-B), 25 for close-set scene text recognition ( IV-C), and 30 for openset scenarios (IV-D) because Chinese and Japanese scripts are generally longer.

Our model is trained from scratch with the same training procedure of the released code 6 of DAN [37]. More specifically, the input image is firstly converted to grayscale. Next, the image is resized to a fixed height of 32 while preserving the aspect ratio until the width exceeded 128 . The model is then optimized with ADADELTA optimizer [45], where the learning rate is set to 1 and drops to 0.1 after three epochs.

\footnotetext{
5 https://github.com/lancercat/OSOCR

${ }^{6}$ https://github.com/Wang-Tianwei/Decoupled-attention-network
} 
TABLE I

ZERO-SHOT CHARACTER RECOGNITION ACCURACY ON HWDB AND CTW DATASETS.

\begin{tabular}{|c|c|c|c|c|c|c|c|c|c|}
\hline \multirow{4}{*}{ Method } & \multirow{4}{*}{ Venue } & \multicolumn{8}{|c|}{ Accuracy (\%) } \\
\hline & & \multicolumn{4}{|c|}{ HWDB } & \multicolumn{4}{|c|}{ CTW } \\
\hline & & \multicolumn{4}{|c|}{ \# characters in training set } & \multicolumn{4}{|c|}{ \# characters in training set } \\
\hline & & 500 & 1000 & 1500 & 2000 & 500 & 1000 & 1500 & 2000 \\
\hline DenseRan [38] & ICFHR'18 & 1.70 & 8.44 & 14.71 & 63.8 & 0.12 & 1.50 & 4.95 & 10.08 \\
\hline FewRan [36] & PRL'19 & 33.6 & 41.5 & 63.8 & 70.6 & 2.36 & 10.49 & 16.59 & 22.03 \\
\hline HCCR [3] & PR'20 & 33.71 & 53.91 & 66.27 & $\overline{73.42}$ & 23.53 & 38.47 & 44.17 & 49.79 \\
\hline Ours & - & 46.67 & 72.19 & 79.82 & 84.31 & 27.94 & 48.23 & 58.56 & 63.77 \\
\hline
\end{tabular}

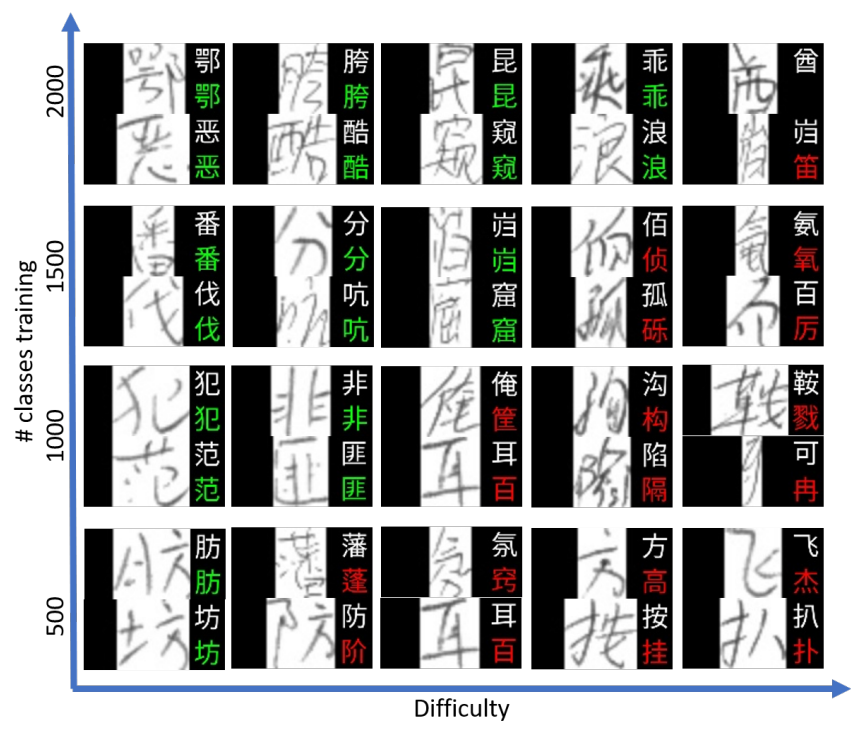

Fig. 6. Zero-shot recognition results on the HWDB dataset: Ground truth is annotated at the upper-right corner and prediction annotated at the bottom-left corner. Wrong predictions are indicated with the red color. Blank prediction indicates the model regards it as an unknown character.

\section{B. Zero-Shot Chinese Character Recognition}

Zero-shot character recognition is a special case of open-set text recognition, where $\mathcal{C}_{\text {train }} \cap \mathcal{C}_{\text {eval }}=\phi$ and the lengths of all samples are equal to one. We validate our method on the Chinese character recognition challenge to provide an intuitive comparison on the ability to handle novel characters against existing zero-shot character recognition methods. We use the two typical datasets, CTW dataset [44] and HWDB [19] dataset, and we split the datasets following the way used in [3] for fair comparisons. Our split is released alongside our codes. The performances on both datasets are shown in Table I

The HWDB dataset is a single-character hand-written dataset collected from 1020 writers. We use HWDB 1.0-1.2 for training and the ICDAR13 competition dataset for evaluation. Same as the strategy in [3], we first randomly split 1000 characters for the evaluation set, and then randomly sample 500, 1000, 1500, and 2000 characters from the remaining characters for training. Examples from the HWDB are shown in Figure 6 The samples are organized to demonstrate what kind of samples are likely to be mistaken.

On this dataset, the model shows robust performance due to no noises in the background. Specifically, the character recognition accuracy (with 500 characters in training) is improved

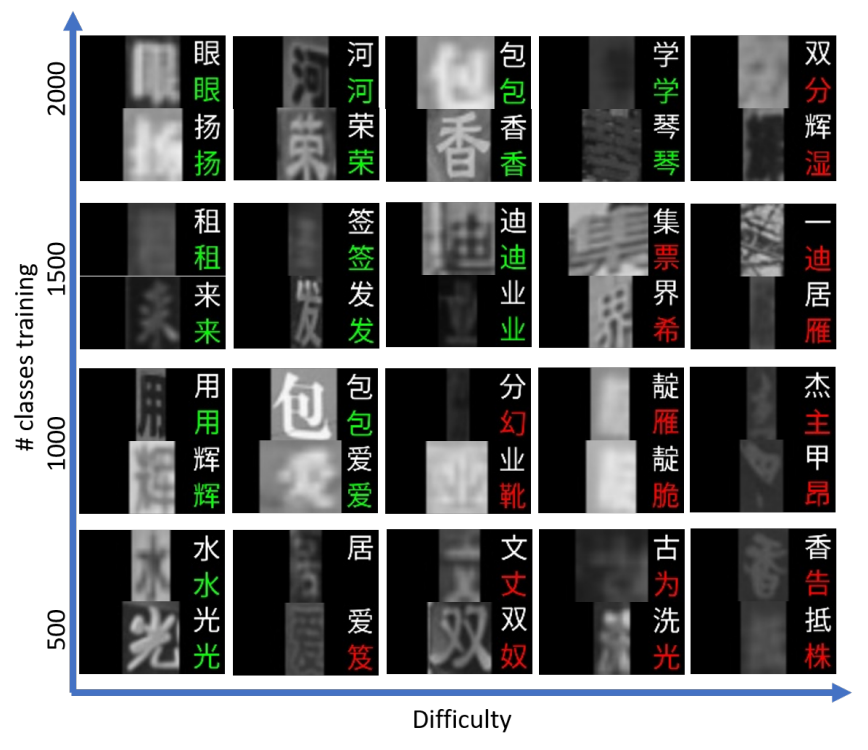

Fig. 7. Zero-shot recognition results on the CTW dataset: Ground truth is annotated at the upper-right corner, and prediction annotated at the bottom-left corner. Wrong predictions are indicated with the red color. Blank prediction indicates the model regards it as an unknown character.

by $12.96 \%$ compared with a recent method [3]. However, the model may fail when a character is written in a cursive way or not correctly written (top-right sample in Figure 6. Also, we can find the model robustness against such patterns improves as the number of training characters increases.

The CTW dataset is a scene text recognition dataset with character-level annotations. This dataset is more difficult than HWDB due to noise, background pattern, and low contrast. Same as the strategy in [3], we first split 500 characters for testing and then randomly sample 500,1000, 1500, and 2000 characters for training. As the 500 characters setup has too few samples, we repeat the training dataset twice to make sure the network goes through enough iterations. Representative samples from the CTW dataset are shown in Figure 7

Experimental results and sample images show that our framework shows robustness against deterioration. Specifically, the character recognition accuracy (with 2000 characters in training) is improved by $13.98 \%$ compared with a recent method [3].

Summarily, our proposed method achieves significant advantages on all datasets and setups against state-of-the-art methods. Our method shows strong generalization ability against novel characters with about $10 \%$ accuracy improve- 
TABLE II

PERFORMANCE ON CONVENTIONAL CLOSE-SET BENCHMARKS, WHERE * INDICATES CHARACTER-LEVEL ANNOTATION REQUIRED

\begin{tabular}{c|c|c|c|ccccc}
\hline Methods & Venue & Training Set & RNN & IIIT5K & SVT & IC03 & IC13 & CUTE \\
\hline AON [4] & CVPR'18 & MJ+ST & Y & 87.0 & 82.8 & 91.5 & - & 76.8 \\
ACE [40] & CVPR'19 & MJ & Y & 82.3 & 82.6 & 89.7 & 82.6 & 82.6 \\
Comb.Best [1] & ICCV'19 & MJ+ST & Y & 87.9 & 87.5 & 94.4 & 92.3 & 71.8 \\
Moran [21] & PR'19 & MJ+ST & Y & 91.2 & 88.3 & 95 & 92.4 & 77.4 \\
SAR [16] & AAAI'19 & MJ+ST & Y & 91.5 & 84.5 & - & - & 83.3 \\
ASTER [29]] & PAMI'19 & MJ+ST & Y & 93.4 & $\mathbf{9 3 . 6}$ & 94.5 & 91.8 & 79.5 \\
ESIR [46] & CVPR'19 & MJ+ST & Y & 93.3 & 90.2 & - & - & 83.3 \\
SCATTER [18] & CVPR'20 & MJ+ST+Extra & Y & 93.7 & 92.7 & $\mathbf{9 6 . 3}$ & $\mathbf{9 3 . 9}$ & $\mathbf{8 7 . 5}$ \\
SEED [26] & CVPR'20 & MJ+ST & Y & 93.8 & 89.6 & - & 92.8 & 83.6 \\
DAN [37]] & AAA'20 & MJ+ST & Y & $\mathbf{9 4 . 3}$ & 89.2 & 95.0 & 93.9 & 84.4 \\
\hline Rosetta [2][1] & KDD'18 & MJ+ST & N & 84.3 & 84.7 & 92.9 & 89.0 & 69.2 \\
CA-FCN* [17] & AAAI'19 & ST & N & 92.0 & 82.1 & - & 91.4 & 78.1 \\
TextScanner* [34] & AAAI'20 & MJ+ST+Extra & N & 93.9 & 90.1 & - & 92.9 & 83.3 \\
\hline Ours & - & MJ+ST & N & 90.40 & 83.92 & 91.00 & 90.24 & 82.29 \\
Ours-Large & - & MJ+ST & N & 92.63 & 88.25 & 93.42 & 93.79 & 86.80 \\
\hline
\end{tabular}

ment on both HWDB and CTW datasets. Moreover, unlike radical-based methods restricted to the Chinese characters, our framework utilizes glyphs from the Noto fonts, which are publicly available and cover most languages. This technique brings two folds of benefits. First, our method does not exploit any language-specific priors, thus not restricted to specific languages. Second, the fonts are easily accessible without extra labeling efforts.

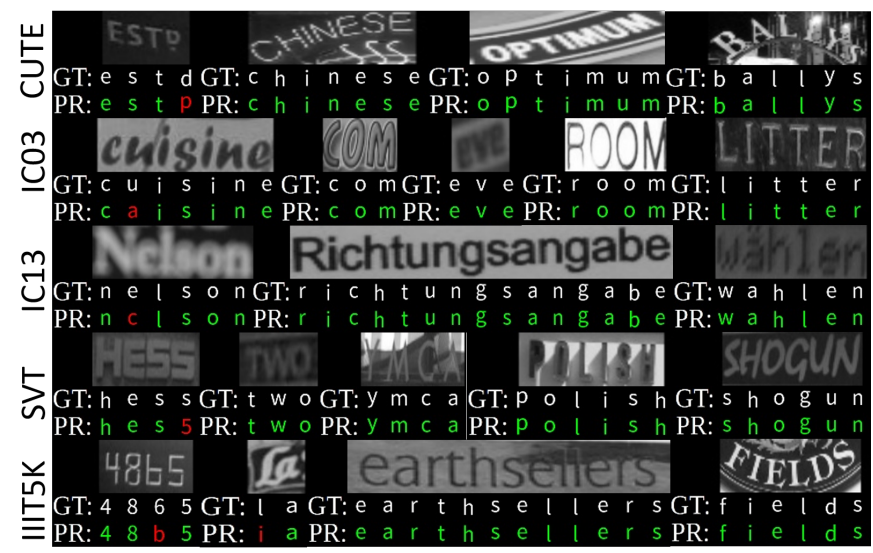

Fig. 8. Recognition examples on the close-set benchmarks: Ground truth is annotated with "GT", prediction is annotated with "PR", and wrong predictions are indicated with the red color.

TABLE III

EXPERIMENTS ON LEXICON-BASED CLOSE-SET BENCHMARKS. $c$ INDICATES CLOSE-SET METHODS AND ${ }^{+}$INDICATES DATASETS OTHER THAN MJ AND ST ARE USED.

\begin{tabular}{l|l|l|l|l}
\hline Method & Venue & $\begin{array}{l}\text { IIIT5k } \\
\text { (small/medium) }\end{array}$ & $\begin{array}{l}\text { IC03 } \\
\text { (full) }\end{array}$ & $\begin{array}{l}\text { SVT } \\
(50)\end{array}$ \\
\hline AON $^{c}$ [4] & CVPR'18 & $99.6 / 98.1$ & 96.7 & 96 \\
ESIR $^{c}$ [46] & CVPR'19 & $99.6 / 98.8$ & - & 97.4 \\
CA-FCN $^{c+}[17]$ & AAAI'19 & $\mathbf{9 9 . 8 / 9 8 . 9}$ & - & $\mathbf{9 8 . 5}$ \\
\hline \hline Zhang et al. [47] & ECCV'20 & $96.2 / 92.8$ & 93.3 & 92.4 \\
\hline Ours & - & $99.53 / 98.63$ & $\mathbf{9 6 . 7 7}$ & 96.75 \\
\hline
\end{tabular}

\section{Standard Close-Set Text Recognition}

Experiments on popular close-set benchmarks are also conducted, and show that our method suffices as a feasible lightweight model on conventional close-set text recognition tasks. Same as the strategies in [37], we train our model on synthetic samples from Jaderberg (MJ) [13] and Gupta (ST) [10], and use the IIIT5K-Words (IIIT5K), Street View Text (SVT), ICDAR 2003 (IC03), ICDAR 2013 (IC13), and CUTE80 (CUTE) as our testing sets. Among the testing sets, IIIT5K, IC03, IC13, and SVT focus on regular shaped texts, and CUTE focuses on irregular shaped text. IIIT5K contains 3,000 testing images collected from the web. SVT has 647 testing images from Google Street View. IC03 includes 867 words from scene text, while IC13 extends IC03 and contains 1015 images. CUTE80 includes 288 curved samples. Our final model is trained for 5 epochs on all these benchmarks.

In the close-set scenarios, our lightweight method is performance-wise comparable to state-of-the-art RNN-free methods, e.g., Rosetta [2] and CA-FCN [17], and also shows an acceptable margin with the newest complicated RNN-based methods. In addition, we also provide a large model trading inference speed for more accuracy. The large model adopts augmentation by $\mathrm{Yu}$ et.al. [43] and enlarges the backbone by 1.5 times. The large model yields close performance to heavy RNN based methods while maintaining a reasonable speed. Note that our methods are RNN-free methods, which does not require batching up to margin out the latency caused by RNNs. Moreover, our method does not require character annotations for training. The detailed results are shown in Table III. We also conduct a dictionary-based test to compare with other methods (Table III), These experiments show that our method exhibits reasonable robustness against the large variety of styles and noises in the real world comparing to Zhang et. al.'s methods [47].

Representative samples are qualitatively illustrated in Fig. 8 Our model demonstrates some extent of robustness for text with blur, irregular-shaped, and different styles. However, due to the absence of RNN that provides modeling for context information, our method shows more tendency to confuse characters that have less distinguishable shapes. 
We also measure the speed of our framework with an RTX2070 Mobile GPU. Models are running with FP32 datatype and the speed is computed by averaging the mean speed overall datasets in Table II Results with different setups are shown in Table IV], where the speeds in [1] are also listed as a rough reference. For maximum throughput, our method can reach a 213 FPS running multi-batched. For latency critique tasks, our method can manage a $9.2 \mathrm{~ms}$ latency under singlebatched mode on the device. Moreover, the experiment shows that topology-preserving transformation in our method has little impact on the inference speed. Space-wise, our models do not require much space either. In summary, our model is reasonably small and fast, thus friendly to smaller devices like laptops, phones, and single-board machines.

TABLE IV

SPEED EVALUATION ON CLOSE-SET BENCHMARKS, WHERE ${ }^{*}$ ' MEANS THE RESULTS ARE DERIVED FROM [1].

\begin{tabular}{|c|c|c|c|c|c|}
\hline Method & $\begin{array}{l}\text { Batch } \\
\text { size }\end{array}$ & $\begin{array}{l}\text { IIIT5K } \\
\text { CUTE }\end{array}$ & GPU & $\begin{array}{l}\text { Speed } \\
\text { (ms/image) }\end{array}$ & $\begin{array}{l}\text { Vram } \\
\text { (MB) }\end{array}$ \\
\hline CRNN* [27] & 1 & $\begin{array}{l}78.2 \\
-\end{array}$ & $\mathrm{P} 40$ & 4.4 & - \\
\hline 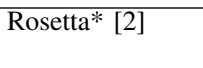 & 1 & $\begin{array}{l}84.3 \\
69.2\end{array}$ & $\mathrm{P} 40$ & 4.7 & - \\
\hline GRCNN* $* 35$ & 1 & $\begin{array}{l}80.8 \\
-\end{array}$ & $\mathrm{P} 40$ & 10.7 & - \\
\hline Comb.Best* & 1 & $\begin{array}{l}87.9 \\
74.0\end{array}$ & $\mathrm{P} 40$ & 27.6 & - \\
\hline Ours w/o TPT & 1 & $\begin{array}{l}89.60 \\
76.04\end{array}$ & RTX2070 & 8.6 & 1161 \\
\hline Ours & 1 & $\begin{array}{l}90.40 \\
82.29\end{array}$ & RTX2070 & 9.19 & 1227 \\
\hline Ours & 16 & $\begin{array}{l}90.40 \\
82.29\end{array}$ & RTX2070 & 4.68 & 1519 \\
\hline Ours-Large & 1 & $\begin{array}{l}92.03 \\
83.68\end{array}$ & RTX2070 & 21.57 & 1377 \\
\hline Ours-Large & 16 & $\begin{array}{l}92.63 \\
86.80 \\
\end{array}$ & RTX2070 & 7.81 & 1663 \\
\hline
\end{tabular}

In conclusion, despite showing an acceptable margin against heavy state-of-the-art methods, our method is comparable or better than RNN-free methods for close-set text recognition. On lexicon-based text recognition experiments, our method shows a significant advantage against the other open-set recognition method [47] on conventional close-set tasks.

\section{Open-Set Text Recognition}

1) Dataset: In the open-set text recognition task, our model is trained on a dataset containing Chinese and Latin samples collected from ART [5], RCTW [30], LSVT [31], CTW [44], and the Latin-Chinese subset of the MLT dataset 7 More specifically, the character set $\mathcal{C}_{\text {train }}$ contains simplified Tier-1 Chinese characters, English letters('A'-' $\mathrm{z}$ '), and digits ('0'-'9'), and samples with characters not covered by $\mathcal{C}_{\text {train }}$ are excluded from the training set. Scripts used to prepare the dataset are released with our codes. The testing dataset contains Japanese samples drawn for the MLT dataset. We use all 1460 characters that appear in this subset as the character set of the testing set $\mathcal{C}_{\text {eval }}$. To simplify the task and focus

${ }^{7}$ Crops with language annotated as Latin and Chinese.
TABLE V

LINE ACCURACY (LA) AND CHARACTER ACCURACY (AR) ON THE OPEN-SET TEXT RECOGNITION DATASET.

\begin{tabular}{c|c|c|c}
\hline Method & CA (\%) & LA (\%) & Speed (ms) \\
\hline Conventional & 38.86 & 17.68 & 5.22 \\
\hline Ours & 47.89 & 29.08 & $\mathbf{4 . 8 2}$ \\
\hline Ours-Large & $\mathbf{4 9 . 1 0}$ & $\mathbf{3 0 . 0 8}$ & 16.30 \\
\hline
\end{tabular}

on the open-set problem, we remove all vertical texts in the training and the testing set. The testing set contains 4,009 text lines.

Because the charsets of Japanese and Chinese languages overlap, the conventional method does not yield a zeroaccuracy. In this set of experiments, we offer a conventional version of our method as a performance reference. The performance is measured in line accuracy (LA) and character accuracy (CA) which are widely used in the OCR community. LA is computed by

$$
L A=\frac{\text { Number of correctly recognized lines }}{\text { Number of lines }},
$$

And CA is defined as

$$
C A=1-\frac{1}{N} \sum_{i}^{N} \frac{\text { EditDistance }\left(P R_{i}, G T_{i}\right)}{\text { length }\left(G T_{i}\right)} .
$$

Here, Ground truth is annotated with "GT", prediction is annotated with "PR", and wrong predictions are indicated with the red color.

2) Results: The experimental results for open-set text recognition are shown in Table $\mathrm{V}$, and some qualitative samples are shown in Figure 9. The conventional method is composed of the recognition backbone used in our framework and a linear classifier for classification. Experiments show that our method yields significantly better performance against the conventional method, in terms of both character accuracy and line accuracy. The significant accuracy gain indicates that our method can handle a reasonable amount of open-set characters, mostly Japanese Kanji characters not included in the simplified Tier-1 Chinese characters. By the way, the accuracy shows a significant margin for single character recognition experiments on the CTW dataset. The margin is caused by the definition of line accuracy, where one wrongly recognized character in a line can lead to a prediction error, resulting in a significantly lower number.

Despite showing reasonable robustness on the unique Kanji in Japanese, our model shows limited generalization on Hiragana and Katakana characters. This can be seen in the breakdown shown in Table VI. In the table, "Sample Requires" indicates each sample must include at least one type of the listed types, while "Sample Excludes" indicates each sample cannot include any of the listed types of characters. "Shared Kanji" indicates Kanji covered by the Tier-1 Simplified Chinese characters [19], while "Unique Kanji" indicates other Kanji that are novel to the model.

3) Limitation: The major problem of our method is to deal with Hiragana and Katakana. Possibly due to Hiragana and Katakana reside in an under-sampled subspace on the 

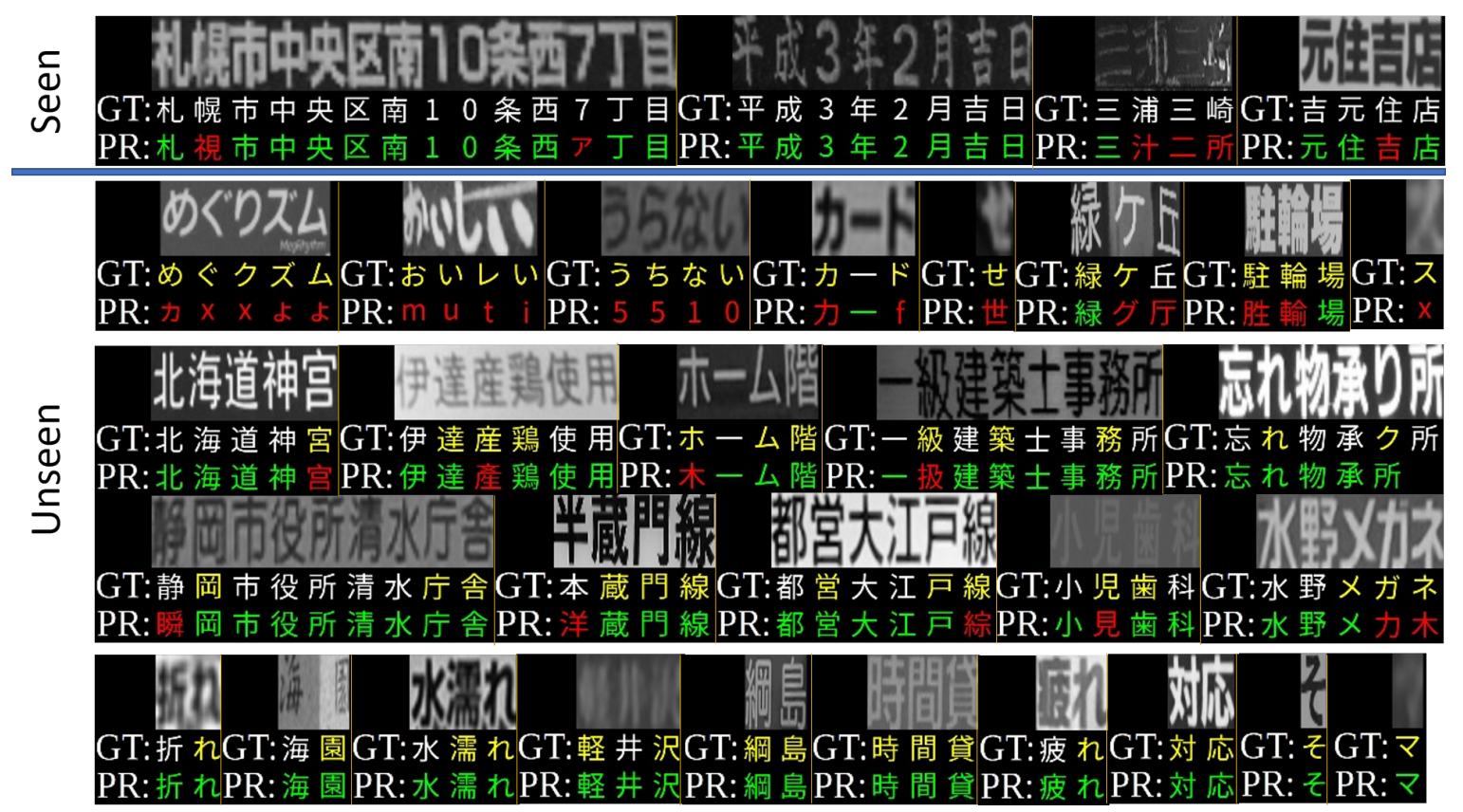

Fig. 9. Good and failed cases in open-set text recognition experiments. Our model works better with shorter scripts and Kanji, while still bad in complex situations with multiple occurrences of Hiraganas and Katakanas. Ground truth is annotated with "GT", prediction is annotated with "PR", and wrong predictions are indicated with the red color. Yellow characters in GT indicate that characters appear in the training set and white indicates seen characters. Correct predictions are indicated with the green color and prediction errors are highlighted with the red color.

TABLE VI

RESULT BREAKDOWN FOR OPEN-SET CHARACTER RECOGNITION.

\begin{tabular}{|c|c|c|c|c|}
\hline Name & $\begin{array}{l}\text { Sample } \\
\text { Requires }\end{array}$ & Sample Excludes & $\mathrm{CA}(\%)$ & $\mathbf{L A}(\%)$ \\
\hline $\begin{array}{l}\text { Shared } \\
\text { Kanji }\end{array}$ & Shared Kanji & $\begin{array}{l}\text { Hiragana, } \\
\text { Katakana, } \\
\text { Unique Kanji }\end{array}$ & 79.86 & 73.81 \\
\hline $\begin{array}{l}\text { Unique } \\
\text { Kanji }\end{array}$ & Unique Kanji & $\begin{array}{l}\text { Hiragana, } \\
\text { Katakana }\end{array}$ & 71.74 & 34.08 \\
\hline All Kanji & $\begin{array}{ll}\text { Unique } & \text { Kanji } \\
\text { or Shared Kanji }\end{array}$ & $\begin{array}{l}\text { Hiragana, } \\
\text { Katakana - }\end{array}$ & 75.33 & 51.64 \\
\hline Kana & $\begin{array}{ll}\text { Hiragana } & \text { or } \\
\text { Katakana } & \end{array}$ & & 18.75 & 0.10 \\
\hline All & & & 47.89 & 29.08 \\
\hline
\end{tabular}

template space $\mathcal{T}$, there are significant visual differences of characters in the training set. This assumption can also be backed by the t-SNE [32] visualization of generated prototypes (shown in Fig. 10p. We can see that Hiragana and Katakana gather at the left-most region of the oval spanned by the training set characters (gray dots), while most other novel characters like traditional characters and Kanji are distributed relatively evenly in the space. Since the network training is a function fitting process, the points from less-sufficiently sampled regions tend to have larger fitting errors. In this case, the under-sampling problem causes bad recognition accuracy.

\section{E. Ablation Study}

To validate the robustness of the proposed modules, we performed extensive ablation studies on all three tasks, i.e., zero-

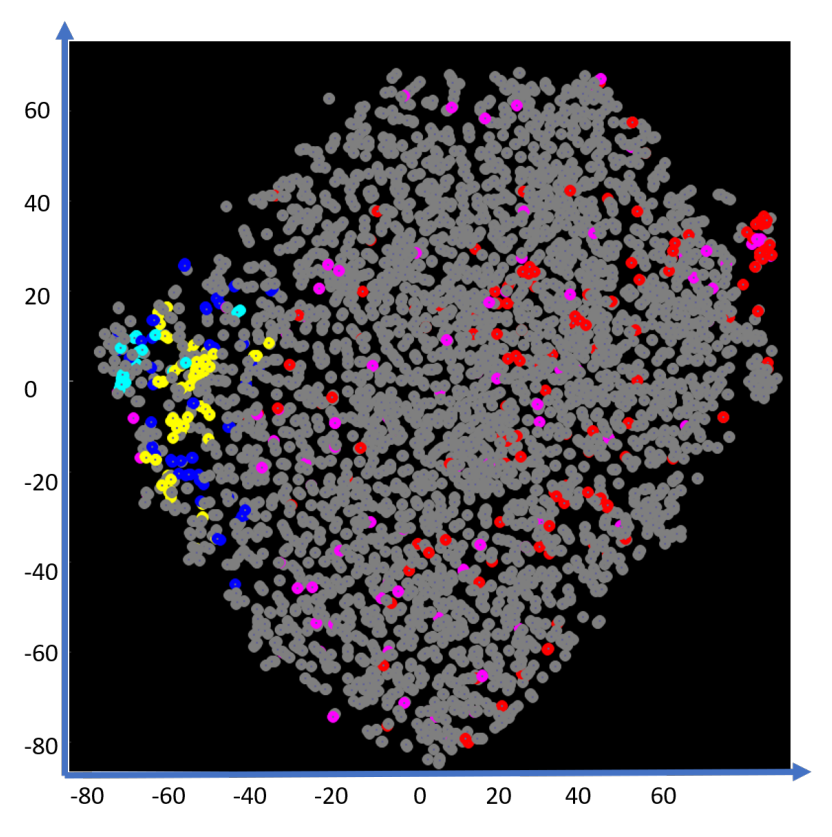

Fig. 10. The t-SNE visualization on ProtoCNN outputs: Gray indicates 3755 simplified Chinese characters and English letters, and red indicates other valid Chinese characters. Purple indicates unique characters in Kanji, blue and yellow for Hiragana and Katakana, and cyan for symbols.

shot Chinese character recognition, close-set text recognition, and open-set text recognition.

We first perform ablation studies on the zero-shot character recognition experiment to validate how $L_{e m b}$ affects the generalization ability of the model. Parameters and set-ups 


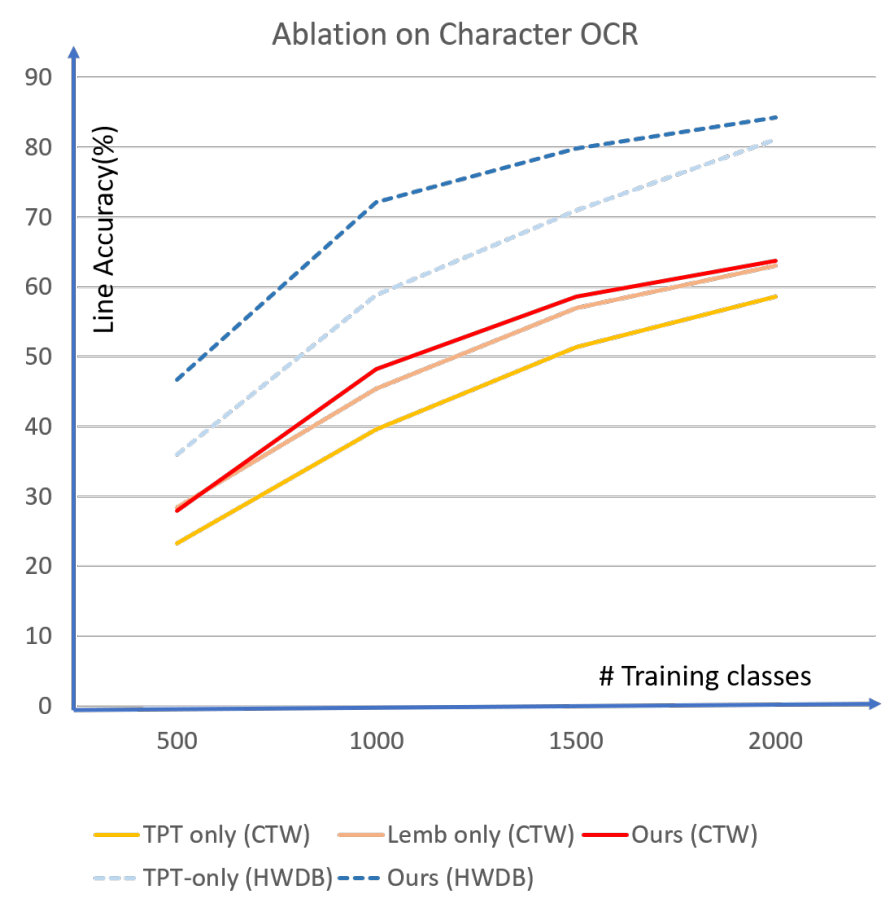

Fig. 11. Effects on $L_{e m b}$ and TPTNet for zero-shot character recognition: Xaxis indicates the number of training classes, and Y-axis indicates recognition accuracy.

are the same as the ones in the above experiments. As the ablation experiments (Fig. 11) show that $L_{e m b}$ improves the performance significantly with less training data. Our assumption is, pushing prototypes apart makes the ProtoCNN more detail-sensitive, hence more robust to confusion potentially caused by characters with similar shape. Additionally, the experiments also show that the topology-preserving transformation provides a limited improvement on scene text character recognition tasks, due to precisely located characters are less prone to geometry deterioration.

We then perform ablation studies to validate the effectiveness of the proposed topology-preserving transformation (TPT) in scene text recognition. Experimental results show that TPT yields a noticeable improvement on most datasets under both normal and large configurations. Noticeably, the TPT yields significant improvement on the CUTE dataset, which contains a lot of irregular shaped text.

TABLE VII

ABLATION STUDIES FOR THE TOPOLOGY PRESERVING TRANSFORMATION ON CONVENTIONAL CLOSE-SET TEXT RECOGNITION BENCHMARKS.

\begin{tabular}{l|l|l|l|l|l}
\hline Method & TPT & $L_{e m b}$ & $\begin{array}{l}\text { IIIT5K } \\
\text { SVT }\end{array}$ & $\begin{array}{l}\text { IC03 } \\
\text { IC13 }\end{array}$ & CUTE \\
\hline Ours-Large & $\checkmark$ & $\checkmark$ & $\mathbf{9 2 . 6 3}$ & $\mathbf{9 3 . 4 2}$ & $\mathbf{8 6 . 6 0}$ \\
& & & $\mathbf{8 8 . 2 5}$ & $\mathbf{9 3 . 7 9}$ & \\
\hline Ours-Large & & $\checkmark$ & 90.90 & 92.73 & 82.63 \\
w/o TPT & & & 85.93 & 90.73 & \\
\hline \hline Ours & $\checkmark$ & $\checkmark$ & 90.40 & 91.00 & 82.29 \\
& & & 83.92 & 90.24 & \\
\hline Ours w/o & & $\checkmark$ & 89.60 & 92.50 & 76.04 \\
TPT & & & 83.46 & 91.03 & \\
\hline
\end{tabular}

Finally, we perform ablation studies on the proposed open-
TABLE VIII

ABLATION STUDY ON THE OPEN-SET TEXT RECOGNITION DATASET.

\begin{tabular}{c|c|c|c}
\hline Method & TPT & $L_{e m b}$ & LA \\
\hline Ours-Large & $\checkmark$ & $\checkmark$ & $\mathbf{3 1 . 0 8}$ \\
Ours-Large w/o TPT & & $\checkmark$ & 27.19 \\
Ours-Large w/o $L_{e m b}$ & $\checkmark$ & & 30.65 \\
\hline Ours & $\checkmark$ & $\checkmark$ & $\mathbf{2 9 . 0 8}$ \\
Ours w/o TPT & & $\checkmark$ & 27.76 \\
Ours w/o $L_{e m b}$ & $\checkmark$ & & 26.29 \\
\hline
\end{tabular}

set text recognition challenge to validate the effectiveness of each module on the proposed task by removing them from the full model. Results are shown in Table VIII. Similarly, both three modules, i.e., topology-preserving transformation (TPT) and the embedding regularization $\left(L_{e m b}\right)$, improve the recognition performance on the openset challenge.

In summary, in our label-to-prototype learning framework, topology-preserving transformation shows robust improvement on all setups in all three tasks. The regularization term $L_{e m b}$ on label-to-prototype learning also shows steady improvements on novel characters, potentially by improving the inter-class distance on the prototype space, leaving more space for novel classes. Because for close-set benchmarks, it doesn't matter whether the prototypes are highly related to shape or not, as long as all known centers distribute far enough with each other. While on the open-set challenge, a less shape-related label-toprototype learning has a higher risk to map novel classes to very close points on the prototype space, which will cause worse classification performance.

\section{CONCLUSIONS}

We propose a novel method for open-set text recognition via label-to-prototype learning. Results show a largely improved performance compared with state-of-the-art methods on zeroshot character recognition tasks. Our framework also reaches competitive performance on the conventional close-set text line recognition benchmarks. Moreover, our method draws a strong baseline on the open-set text recognition task with a large character set and complex deterioration. In summary, our method can robustly handle isolated characters and text lines in both open-set and close-set scenarios.

Despite showing impressive generalization capability on open-set scenarios, our method still has some limitations. Specifically, our method tends to fail to recognize characters that have significantly different shapes from the seen characters (e.g., Hiragana and Katakana). The shape difference results in these characters residing in the under-sampled regions on the prototype space not well-covered by the seen characters, causing lower recognition accuracy. Domain transferring may be investigated to solve the domain bias among complex characters and different languages in the future research.

\section{ACKNOWLEDGEMENT}

The research is supported by the National Key Research and Development Program of China (2020AAA09701) and the National Natural Science Foundation of China (62006018). 


\section{REFERENCES}

[1] Jeonghun Baek, Geewook Kim, and Junyeop Lee et. al. What is wrong with scene text recognition model comparisons? dataset and model analysis. In ICCV, pages 4714-4722, 2019.

[2] Fedor Borisyuk, Albert Gordo, and Viswanath Sivakumar. Rosetta: Large scale system for text detection and recognition in images. In $K D D$, pages 71-79, 2018

[3] Zhong Cao, Jiang Lu, Sen Cui, and Changshui Zhang. Zero-shot handwritten chinese character recognition with hierarchical decomposition embedding. Pattern Recognition, 107:107488, 2020.

[4] Zhanzhan Cheng, Yangliu Xu, and Fan Bai et.al. AON: towards arbitrarily-oriented text recognition. In CVPR, pages 5571-5579, 2018.

[5] Chee Kheng Chng, Errui Ding, and Jingtuo Liu et. al. ICDAR2019 robust reading challenge on arbitrary-shaped text - rrc-art. In ICDAR, pages 1571-1576. IEEE, 2019.

[6] Jifeng Dai, Haozhi Qi, and Yuwen Xiong et. al. Deformable convolutional networks. In ICCV, pages 764-773. IEEE Computer Society, 2017.

[7] Titir Dutta, Anurag Singh, and Soma Biswas. Adaptive margin diversity regularizer for handling data imbalance in zero-shot SBIR. In ECCV, volume 12350 of Lecture Notes in Computer Science, pages 349-364. Springer, 2020.

[8] Spyros Gidaris and Nikos Komodakis. Dynamic few-shot visual learning without forgetting. In $C V P R$, pages 4367-4375. IEEE Computer Society, 2018.

[9] Spyros Gidaris and Nikos Komodakis. Generating classification weights with GNN denoising autoencoders for few-shot learning. In $C V P R$, pages $21-30,2019$.

[10] Ankush Gupta, Andrea Vedaldi, and Andrew Zisserman. Synthetic data for text localisation in natural images. In CVPR, pages 2315-2324, 2016.

[11] Bharath Hariharan and Ross B. Girshick. Low-shot visual recognition by shrinking and hallucinating features. In ICCV, pages 3037-3046. IEEE Computer Society, 2017.

[12] Kaiming He, Xiangyu Zhang, and Shaoqing Ren et. al. Deep residual learning for image recognition. In CVPR, pages 770-778, 2016.

[13] Max Jaderberg, Karen Simonyan, and Andrea Vedaldi et. al. Synthetic data and artificial neural networks for natural scene text recognition. CoRR, abs/1406.2227, 2014.

[14] Siwei Lai, Liheng Xu, and Kang Liu et. al. Recurrent convolutional neural networks for text classification. In Blai Bonet and Sven Koenig, editors, Proceedings of the Twenty-Ninth AAAI Conference on Artificial Intelligence, January 25-30, 2015, Austin, Texas, USA, pages 22672273. AAAI Press, 2015.

[15] Junyeop Lee, Sungrae Park, and Jeonghun Baek et. al. On recognizing texts of arbitrary shapes with $2 \mathrm{~d}$ self-attention. In $C V P R$, pages $2326-$ 2335. IEEE, 2020.

[16] Hui Li, Peng Wang, and Chunhua Shen et. al. Show, attend and read: A simple and strong baseline for irregular text recognition. In $A A A I$, pages 8610-8617, 2019.

[17] Minghui Liao, Jian Zhang, and Zhaoyi Wan et. al. Scene text recognition from two-dimensional perspective. In AAAI, pages 8714-8721, 2019.

[18] Ron Litman, Oron Anschel, and Shahar Tsiper et. al. SCATTER selective context attentional scene text recognizer. In $C V P R$, pages 11959-11969, 2020

[19] Cheng-Lin Liu, Fei Yin, and Da-Han Wang et.al. CASIA online and offline chinese handwriting databases. In ICDAR, pages 37-41. IEEE Computer Society, 2011.

[20] Jiang Lu, Pinghua Gong, and Jieping Ye et. al. Learning from very few samples: A survey. CoRR, abs/2009.02653, 2020.

[21] Canjie Luo, Lianwen Jin, and Zenghui Sun. MORAN: A multiobject rectified attention network for scene text recognition. Pattern Recognition, 90:109-118, 2019.

[22] Tristan Postadjian, Arnaud Le Bris, and Clément Mallet et. al. Superpixel partitioning of very high resolution satellite images for large-scale classification perspectives with deep convolutional neural networks. In IGARSS, pages 1328-1331, 2018.

[23] Farhad Pourpanah, Moloud Abdar, and Yuxuan Luo et.al. A review of generalized zero-shot learning methods. CoRR, abs/2011.08641, 2020.

[24] Hang Qi, Matthew Brown, and David G. Lowe. Low-shot learning with imprinted weights. In CVPR, pages 5822-5830. IEEE Computer Society, 2018.

[25] Siyuan Qiao, Chenxi Liu, and Wei Shen et. al. Few-shot image recognition by predicting parameters from activations. In $C V P R$, pages 7229-7238. IEEE Computer Society, 2018.

[26] Zhi Qiao, Yu Zhou, and Dongbao Yang et. al. SEED: semantics enhanced encoder-decoder framework for scene text recognition. In CVPR, pages 13525-13534, 2020.
[27] Baoguang Shi, Xiang Bai, and Cong Yao. An end-to-end trainable neural network for image-based sequence recognition and its application to scene text recognition. IEEE Trans. Pattern Anal. Mach. Intell., 39(11):2298-2304, 2017.

[28] Baoguang Shi, Xinggang Wang, and Pengyuan Lyu et. al. Robust scene text recognition with automatic rectification. In CVPR, pages 4168 4176, 2016.

[29] Baoguang Shi, Mingkun Yang, and Xinggang Wang et. al. ASTER: an attentional scene text recognizer with flexible rectification. IEEE Trans. Pattern Anal. Mach. Intell., 41(9):2035-2048, 2019.

[30] Baoguang Shi, Cong Yao, and Minghui Liao et. al. ICDAR2017 competition on reading chinese text in the wild (RCTW-17). In ICDAR, pages 1429-1434. IEEE, 2017.

[31] Yipeng Sun, Dimosthenis Karatzas, and Chee Seng Chan et. al. ICDAR 2019 competition on large-scale street view text with partial labeling RRC-LSVT. In ICDAR, pages 1557-1562. IEEE, 2019.

[32] Laurens Van der Maaten and Geoffrey Hinton. Visualizing data using t-sne. Journal of machine learning research, 9(11), 2008.

[33] Oriol Vinyals, Charles Blundell, and Tim Lillicrap et. al. Matching networks for one shot learning. In Advances in Neural Information Processing Systems 29: Annual Conference on Neural Information Processing Systems 2016, December 5-10, 2016, Barcelona, Spain, pages 3630-3638, 2016.

[34] Zhaoyi Wan, Minghang He, and Haoran Chen et. al. Textscanner: Reading characters in order for robust scene text recognition. In $A A A I$, pages $12120-12127,2020$.

[35] Jianfeng Wang and Xiaolin Hu. Gated recurrent convolution neural network for OCR. In Isabelle Guyon, Ulrike von Luxburg, and Samy Bengio, editors, Advances in Neural Information Processing Systems 30: Annual Conference on Neural Information Processing Systems 2017, 4-9 December 2017, Long Beach, CA, USA, pages 335-344.

[36] Tianwei Wang, Zecheng Xie, and Zhe Li et. al. Radical aggregation network for few-shot offline handwritten chinese character recognition. Pattern Recognition Letters, 125:821-827, 2019.

[37] Tianwei Wang, Yuanzhi Zhu, and Lianwen Jin et. al. Decoupled attention network for text recognition. In AAAI, pages 12216-12224, 2020.

[38] Wenchao Wang, Jianshu Zhang, and Jun Du et. al. Denseran for offline handwritten chinese character recognition. In ICFHR, pages 104-109, 2018.

[39] Yu-Xiong Wang, Ross B. Girshick, and Martial Hebert et. al. Lowshot learning from imaginary data. In CVPR, pages 7278-7286. IEEE Computer Society, 2018.

[40] Zecheng Xie, Yaoxiong Huang, and Yuanzhi Zhu et. al. Aggregation cross-entropy for sequence recognition. In $C V P R$, pages 6538-6547, 2019.

[41] Mingkun Yang, Yushuo Guan, and Minghui Liao et.al. Symmetryconstrained rectification network for scene text recognition. In $C V P R$, pages 9146-9155, 2019.

[42] Han-Jia Ye, Hexiang Hu, and De-Chuan Zhan et. al. Few-shot learning via embedding adaptation with set-to-set functions. In $C V P R$, pages 8805-8814. IEEE, 2020.

[43] Deli Yu, Xuan Li, and Chengquan et. al. Towards accurate scene text recognition with semantic reasoning networks. In CVPR, pages 12110 $12119,2020$.

[44] Tai-Ling Yuan, Zhe Zhu, and Kun Xu et. al. A large chinese text dataset in the wild. J. Comput. Sci. Technol., 34(3):509-521, 2019.

[45] Matthew D. Zeiler. ADADELTA: an adaptive learning rate method. CoRR, abs/1212.5701, 2012.

[46] Fangneng Zhan and Shijian Lu. ESIR: end-to-end scene text recognition via iterative image rectification. In CVPR, pages 2059-2068, 2019.

[47] Chuhan Zhang, Ankush Gupta, and Andrew Zisserman. Adaptive text recognition through visual matching. In $E C C V$, volume 12361 of Lecture Notes in Computer Science, pages 51-67, 2020. 